occasionally tell in the right direction. Had Father Gerard not sacrificed his position by aiming so much at smart writing-had he favoured us with more solid thought instead of endeavouring "to split the ears of the groundlings"-his lucubrations would have received more respectful attention. But satire and cynicism, interspersed with ridicule, are not the best methods for securing consideration from men of science, and it is surprising that the author should have resorted so largely to their use.

R. Meldola.

\section{THE LAWS OF FORCE AND MOTION.}

The Laws of Force and Motion. By John Harris (Kuklos). (London: Wertheimer, Lea, and Co., I 890.)

$\mathrm{N}$ his preface the author, very rightly, sounds a warning note against the arrogance of Conventional Science, in its tendency to become ultra-conservative, intolerant, and extremely dogmatic.

But Real Science will always welcome and encourage attack and contradiction, feeling sure that Truth will ultimately prevail in the consensus of the majority who have devoted themselves dispassionately to the consideration of the facts in dispute. "Transibunt multi et augebit Scientia."

We presume the author would not ask to be judged with more leniency than he has displayed for the opponents he has singled out; so we may say at once that, after careful winnowing, we have not secured those grains of fact and truth which we were led to expect.

The experimental apparatus described seems carefully constructed and suitable for exact measurements; but does not differ essentially from that employed by Smeaton more than Ioo years ago. However, the author assumes the true scientific sceptical spirit, in refusing to accept implicitly the statement of theoretical laws without putting them to the test of practical experimental verification.

Mathematicians will understand the nature of the author's attacks on Conventional Science from the specimen on p. $3 \mathrm{I}$ :-

"It would seem that, some time ago, a highly influential party of natural philosophers (Leibnitz, the two Bernoullis, \&c.) entertained and supported the idea that the momentum of a moving body varies as the square of the velocity. This idea or conclusion was probably based on an inference, that, since a double velocity of the resistance required four times the force to produce it, four times the momentum must have been imparted to the resistance."

After this wavering as to the meaning of momentum, we are quite prepared to find (p. 6o) that the author is of the school who declare that the moon does not rotate.

The author cannot decide between 16.1 or 32.2 for the value of $g$ (p. 24); and cannot settle in consequence whether the normal acceleration in a circle is the squared velocity divided by the radius or by the diameter (p. I9).

"Tangential force" is, in the author's opinion, a more correct scientific term to use than "centrifugal force," although he allows that the latter is hallowed by long usage; but in his treatment he enunciates a theorem on p. 2I, "The actual lineal ratio of the sine to the arc, when the arc is an octant, is 9 to I0," quoted from his own s' Treatise on the Circle and Straight Line"; this makes NO. I I 4 I, VOL. 44 ] $\pi=2 \sqrt{ } 2 \div 0^{\circ} 9$, a result worth recording by collectors of mathematical curiosities.

We hoped to find something combative in the articles on the Tidal Effect of Lunar Gravitation (p. 57), and on the Moon's Gravitative Influence at the Equatorial Surface of the Earth measured by Pendulum Oscillations (p. 76), considering that even the great Abel went astray in his theory at this point; but our author confines himself to vague generalities.

He would perform a valuable service to Science if he employed his experimental skill in observing the effect of Lunar Gravity on the Seconds Pendulum, as Conventional Science asserts that this effect does not amount to more than a rate of one 2ooth of a second in the day, although so noticeable in the Tides.

"Some Propositions in Geometry," by the same author, is advertised at the end of the book, whereof the Trisection of the Angle, the Duplication of the Cube, and the Quadrature and Rectification of the Circle, occupy the chief part; but we wonder whether the author has quite settled in his Geometry that the versed sine (or vertical height) is proportional to the chord, in a circle (p. 7I). This might have been a misprint, but that the author adds immediately a numerical illustration, by saying that, if the chord is duplicated, the versed sine is also duplicated.

And this homely mode of verifying a law of comparison, by halving or doubling some quantity, and then observing the consequent change in the phenomena, is the single idea we consider worth lifting from the book, for general purposes of convincing argument and illustration of a mathematical law.

A. G. G.

\section{OUR BOOK SHELF.}

An Introduction to the Mathematical Theory of Electricity and Magnetism. By W. T. A. Emtage, M.A. (Oxford: Clarendon Press, 1891.)

THE want of a text-book especially designed for the use of candidates for examinations in which a knowledge of the more elementary portions of the mathematical theory of electricity and magnetism is demanded has been felt for some time. Though the absence of such a book has caused some inconvenience, we are not at all sure that it has been detrimental to the study of electricity, for hitherto the candidate for a mathematical examination in electricity has been compelled to learn the subject from books such as those of Maxwell, or of Mascart and Joubert, in which electricity is treated as what it really is outside the examination-room-a subject in which mathematics and experiment are closely mixed and mutually helpful : it is to this that, we think, is to be ascribed a good deal of that interest which electricity, above all other subjects, seems to excite in its students. When, however, the analytical parts of the subject are divorced from the experimental, we do not believe they will be found to excite any special enthusiasm, or that the result will be much more interesting than an ordinary text-book for the Mathematical Tripos on, say, hydrostatics.

There is no doubt, however, that there is a demand for a text-book suitable for examination purposes, and this demand will, we think, be well met by the book before us. The scope of the work may be described by saying that it includes nearly all the analytical parts of Maxwell's larger treatise which do not involve analysis higher than the simpler parts of the differential and integral calculus; 